\title{
Políticas de desarrollo rural y su impacto en la región Sur Oriente de Guatemala
}

\author{
Ariel Oswaldo Reyes Donis \\ arielreyes07@hotmail.com \\ Ingeniero Agrónomo, Maestro en Docencia Universitaria con énfasis en Andragogía y \\ Estudiante del Doctorado en Investigación para el Desarrollo Social \\ Centro Universitario de Sur Oriente, CUNSURORI \\ Universidad de San Carlos de Guatemala
}

Fecha de recepción: 21/05/2018

Fecha de aceptación: 27/07/2018

\begin{abstract}
Resumen
El desarrollo inclusivo, debe surgir como apoyo a las comunidades de las áreas rurales, para que estas puedan incorporarse en los procesos empresariales, contribuyendo así a la reducción de las desigualdades y a la salida de la pobreza de manera estructural, por la vía de mejorar los ingresos y las oportunidades de alcanzar un adecuado estilo de vida, esta visión del desarrollo inclusivo es particularmente importante en la áreas rurales del Suroriente de Guatemala, en donde las desigualdades individuales y territoriales tienden a acentuarse. El apoyo proporcionado por el gobierno de Guatemala, en relación con políticas claras para el fortalecimiento y esclarecimiento de bases legales sólidas, les debe permitir a los pobladores de esta región implementar y participar de manera efectiva en el desarrollo económico del sector agroindustrial del país. En este artículo, se pretende abordar las políticas de desarrollo rural de Guatemala, para analizar si estas permiten una inclusión libre y activa de los pequeños agricultores en el proceso ya mencionado, considerando para el efecto las siguientes interrogantes: ¿son en verdad las políticas de desarrollo agrícola de Guatemala incluyentes para los pequeños productores de las áreas rurales? ¿Cuáles son los factores que afectan negativamente la viabilidad de los pequeños productores del área rural?
\end{abstract}

\section{Palabras clave}

Desarrollo rural, situación agraria, inclusión, cadena de valor, política rural

\begin{abstract}
Inclusive development must emerge as support to the communities of rural areas, so that they can be incorporated into business processes, thus contributing to the reduction of inequalities and the exit of poverty in a structural manner, by way of improving Income and opportunities to achieve an adequate lifestyle, this vision of inclusive development is particularly important in the rural areas of southeastern Guatemala, where individual and territorial inequalities tend to accentuate. The support provided by the Guatemalan government, in relation to clear policies for the strengthening and clarification of solid legal bases, should allow the inhabitants of this region to implement and participate effectively in the economic development of the country's agroindustrial sector. In this article, it is intended to address the rural development policies of Guatemala, to analyze if these allow a free and active inclusion of small farmers in the process, considering for its effect the following questions: are they really the Guatemala's agricultural development policies that are inclusive for small producers in rural areas? What are the factors that negatively affect the viability of small producers in rural areas?
\end{abstract}

\section{Keywords}

Rural development, agrarian situation, inclusion, value chain, rural policy 


\section{Introducción}

En Guatemala el desarrollo rural persigue dar respuesta a tres necesidades básicas para hacer posible un futuro sostenible de nuestra especie: 1. mejorar la formación y el bienestar de los miles de personas que viven en este medio, erradicando la pobreza extrema y evitando su migración hacia la marginación de las ciudades, 2. lograr una producción agrícola sostenible para asegurar que todos los seres humanos tengan acceso a los alimentos que necesitan, 3. proteger y conservar la capacidad de la base de recursos naturales para seguir proporcionando servicios de producción, ambientales y culturales.

La Política de Desarrollo Rural, toma como punto de partida la importancia de conocer las problemáticas, potencialidades, así como el papel económico de estas, persiguiendo el desarrollo humano de las poblaciones que lo habitan, considerando los niveles de desempleo y niveles de crisis a nivel nacional, pero que inciden de manera contundente en el deterioro de las condiciones de vida de las poblaciones que conforman estas áreas.

Por tal motivo se debe conocer el impacto que estas tienen, en el desarrollo inclusivo, de las comunidades de las áreas rurales, para que estas puedan incorporarse en los procesos empresariales, contribuyendo así a la reducción de las desigualdades y a la salida de la pobreza de manera estructural, por la vía de mejorar los ingresos y las oportunidades de alcanzar un adecuado estilo de vida, esta visión del desarrollo inclusivo es particularmente importante en las áreas rurales del Suroriente de Guatemala, en donde las desigualdades individuales y territoriales tienden a acentuarse y en las que el acceso a mercados se hace más costoso.

\section{Marco teórico}

La situación agraria ha sido el eje sobre el que se ha construido la historia de Guatemala y quizás la principal fuente de conflictos políticos, económicos y sociales. Según (Cambranes, 1992), la reforma agraria fue el principal programa de la revolución de 1944 y una de las razones de la intervención es- tadounidense en 1954. Después de una prolongada negociación, la situación agraria fue uno de los temas centrales en los Acuerdos de Paz de 1996. $Y$ hoy, el desarrollo rural y las luchas campesinas están presentes en el debate nacional.

\subsection{Desarrollo rural en Guatemala}

Según Bonilla (2017), la expresión desarrollo rural hace referencia a acciones e iniciativas llevadas a cabo para mejorar la calidad de vida de las comunidades no urbanas. El desarrollo rural ha sido un tema importante de discusión, pues es tocado por diferentes sectores sociales como, políticos, productores, académicos, así como organismos de cooperación internacional, debido a que este es considerado como un eje estratégico de desarrollo humano.

En los últimos años en Guatemala se ha incrementado enormemente la inversión en el área rural mediante la implementación de programas sociales o de asistencia alimentaria, invirtiendo recursos con fines políticos y clientelares, por lo que se considera que estos programas no reflejan una disminución de la pobreza en el área rural ni una mejora en la calidad de vida de las familias beneficiadas ya que no se fomenta la productividad, la sostenibilidad ni la tecnificación. Dentro de estos mencionamos las transferencias monetarias, el programa de bosques y agua para la concordia (Echeverri, 2002:26)

De acuerdo con los datos obtenidos del (INE, 2015), las desigualdades entre el campo y la ciudad constituyen un obstáculo importante al desarrollo sostenible. Algunos indicadores dan cuenta sobre la importancia de este fenómeno en la realidad nacional. Por ejemplo, actualmente el 52\% de la población es rural, hay un $76.4 \%$ de pobreza general rural, existe un $65.1 \%$ de empleo rural y el PIB agrícola representa un $13 \%$.

\subsection{Política de desarrollo rural}

De acuerdo con Contreras (2001), los objetivos de las políticas de desarrollo rural, tienen que ver con la percepción de la realidad en la cual se vive en 
Guatemala, y con el nivel de conocimiento general el cual se tiene sobre esta. Un nivel diferenciado de conocimiento sobre la realidad, genera el retraso de las posibles soluciones al problema, pues se debe comenzar equiparando los conocimientos para generar una propuesta de consenso.

En la política de desarrollo rural de Guatemala, se estableció como uno de sus objetivos, impulsar procesos de transformación y modernización de la agricultura incrementando la producción, productividad y rentabilidad, mejorando de esta manera la competitividad de las empresas en mercados nacionales e internacionales, de manera paralela a esta visión de transformación de una sociedad tradicional hacia una moderna, las organizaciones sociales campesinas, en abril del 2008, instalaron un diálogo por el desarrollo integral y la resolución de la conflictividad agraria, laboral y ambiental.

Según el IPES (2000), desde el punto de vista de la gobernabilidad, debemos tener presente que la población rural es un sujeto sociopolítico que juega un rol determinante en la política, economía, cultura, ideología de contrapoder y resiliente ante las políticas oligopólicas del sistema capitalista.

\subsection{Características de la región Sur Oriente de Guatemala}

La región IV de Guatemala, conocida como la región Sur Oriente, posee características únicas en cuanto a la distribución de sus recursos forestales, pecuarios, agrícolas, extracción y actividades hidroeléctricas; pero se debe ir más a fondo en cuanto a descripción de las características y condiciones específicas del lugar se debe considerar que esta región pertenece al corredor seco de Guatemala.

De acuerdo con Cambranes (1992), un porcentaje importante de la población no posee tierra para trabajarla directamente, por lo que se emplea en la producción de artesanías, en el sector informal de ventas, asalariado agrícola o bien emigra a la ciudad ante falta de oportunidades, los agricultores se dedican a la producción de granos básicos y productos pecuarios, que son utilizados para consumo familiar y comercialización, con poca diversificación por la falta de trasformación por medio de procesos agroindustriales, el acceso a la educación es limitado, así como el desarrollo de las comunidades en términos de infraestructura y economía de sus pobladores.

\subsection{Impacto de la política de desarrollo rural}

Es importante indicar que los cambios estructurales que se han realizado en los últimos años en Guatemala, siguiendo las políticas de desarrollo y los cambios que exigen los procesos de globalización, han generado una brecha significativa entre las diferentes clases sociales (ricos y pobres), esto derivado del interés que presentan los gobiernos de turno, por fortalecer las negociaciones de mercado internacionales, descuidando la implementación de herramientas efectivas y fundamentales que permiten el eficiente desarrollo de las comunidades del área rural, y el Sur Oriente de Guatemala no es exento de esta situación, los indicadores aportados por el INE, muestran la situación de las poblaciones rurales de Guatemala, como se observa en la gráfica 1. 


\section{Gráfica 1 \\ Incidente de pobreza total por área de residencia -República de Guatemala, Encovi 2000, 2006 y 2014, en porcentaje-}

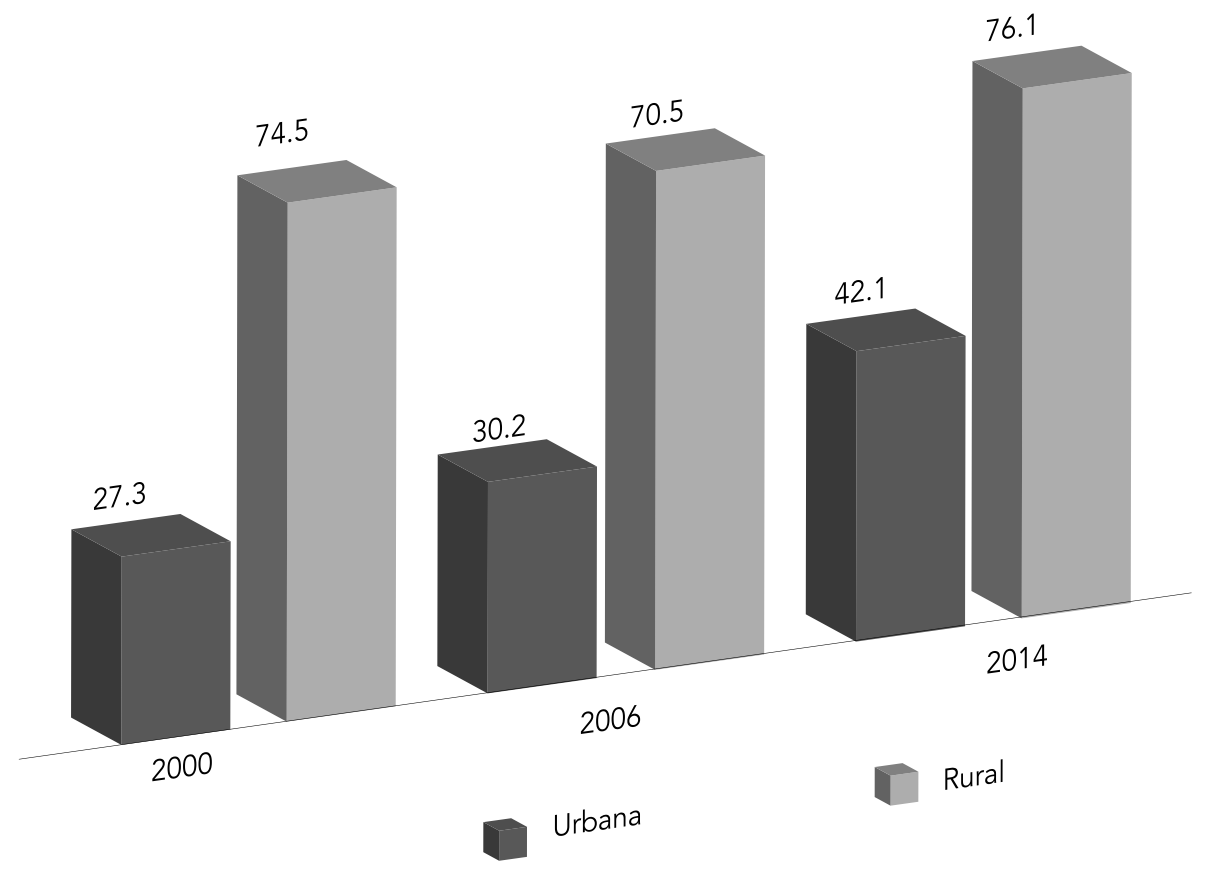

Fuente: Instituto Nacional de Estadística.

Tomando en cuenta que uno de los objetivos de la política de desarrollo rural, es el de impulsar la producción y productividad de los productores de las áreas rurales, se puede decir que, el desarrollo rural por medio de la agroindustria y su vinculación a las cadenas agroalimentarias más complejas son fundamentales como instrumento generador de fuentes adicionales de ingresos en el sector, viabilizando la incorporación de todos los actores sociales, a los frutos del desarrollo económico, es decir, mujeres, jóvenes y pobladores en general, jugando todos estos un papel importante como motor de procesos mayores de desarrollo.

\section{Metodología}

Se procedió a realizar una revisión preliminar del tema, para abordar la temática del impacto de las políticas de desarrollo rural en el sur oriente de
Guatemala. Se analizó la información obtenida, haciendo una sistematización de la misma para concretar la inclusión de los productores rurales derivado de la implementación de dichas políticas. Se hizo un acercamiento a instituciones como el INE y SEGEPLAN para obtener información pertinente en relación al tema.

\section{Resultados}

Considerando lo descrito anteriormente, es de considerar que parte de la desestimulación que presentan los habitantes de las áreas rurales del Sur Oriente de Guatemala, se debe a que las mismas políticas de desarrollo rural actuales, debido a la falta de tierras propias y que estas se centran básicamente en la producción de productos de exportación tradicionales y productos exportables no tradicionales, considerando las condiciones de los 
mercados internacionales, por tal motivo los productores de las áreas rurales no pueden competir debido a la falta de tecnificación de sus procesos de producción.

Como elemento que incide en la viabilidad de los procesos de producción en las áreas rurales de Guatemala, podemos mencionar la falta de tierras propias por parte de los pobladores, así como los programas de asistencia social cuyo auge se dio en el Gobierno de Álvaro Colom, que en la actualidad han generado la dependencia de los pobladores de estas áreas a los diferentes insumos y ayudas económicas obtenidas como beneficio de estos, causando un desequilibrio en las actividades de producción de los pobladores, pues han dejado de ser productivos en su mayoría.

De todo esto se puede decir que las políticas de desarrollo rural van encaminadas al fortalecimiento de las áreas rurales del país, sin considerar las características de cada una de las regiones que lo conforman, por tal motivo se puede entender que estas políticas pueden ser excluyentes, porque aísla al pequeño productor en términos de transferencia de tecnologías, que les permitan participar de manera eficiente en los procesos de producción.

Guatemala es considerado como un país con vocación agrícola, pero se debe considerar que no se están aprovechando las oportunidades y gran potencial que existen; tomando en cuenta que la Región IV área Sur Oriente de Guatemala, posee características adecuadas para el desarrollo de actividades productivas. Al analizar el proceso agroindustrial como una alternativa de estimulación para el desarrollo de las áreas rurales, es necesario tener presente, que el desarrollo de la agricultura rural o campesina, es un problema complejo.

La modernización integral de la economía campesina no puede hacerse de manera aislada de las dinámicas históricas en torno al desarrollo rural y las variantes adaptativas de la agricultura familiar, sobre todo por la desventaja de competir ante los monocultivos agroindustriales e industria extrac- tiva; y reconociendo que los territorios indígenas son bastiones de identidad, lucha comunitaria e inclusión agraria.

\section{Referencias}

Bonilla, G. (2017). Desarrollo Rural Sustentable en Guatemala, Jalapa (Guatemala): SERVIPRENSA

Cambranes, J. C. (1992). Quinientos años de lucha por la tierra. Guatemala (Guatemala): USAC.

Contreras, A. (2001) Ensayos sobre cuestión Agraria. México (DF), Editorial Universitaria.

Del Valle, C. (2001) Derecho agrario y política agraria en Guatemala. Guatemala: Ed. Transgrafic

Echeverri, R. y M. Ribero (2002). Nueva ruralidad, Visión del Territorio en América Latina y el Caribe, San José (Costa Rica): IICA.

FAO (Organización de las Naciones Unidas para la Alimentación y la Agricultura). (2003). Tesauro plurilingüe de tierras. Roma (Italia): Departamento Económico y Social.

FAO (Organización de las Naciones Unidas para la Alimentación y la Agricultura) (2001). Agricultura contractual: Departamento Económico y Social.

Guatemala, Gobierno de la República de. (2009). Política nacional de desarrollo rural integral -PNDRI-: Guatemala (Guatemala), s.e

Instituto Nacional de Estadística INE. (2015). República de Guatemala, Encuesta Nacional Agropecuaria 2014. Guatemala (Guatemala): Xelatex

IPES (Instituto de Estudios Políticos, Económicos y Sociales) (2000). Propuesta de Desarrollo Rural en Guatemala. 
Peñalonzo, V. (2009). "Buena práctica agrícola comunitaria" Manual de buenas prácticas, serie Agroindustrias, pp 1-46

RAE (Real Academia Española). (2001). Diccionario de la lengua española (DRAE). España
Waissbluth, M (1989) "Agroindustria, tecnología doméstica y empleo". Lecturas Seleccionadas sobre Agroindustrias IICA, pp 25-27 